\title{
MitraClip for mitral valve regurgitation and transcatheter aortic valve implantation for severe aortic valve stenosis: state-of-the-art
}

\author{
Tomasz Tokarek ${ }^{1,2}$, Artur Dziewierz ${ }^{1,3}$, Dariusz Dudek ${ }^{3}$ \\ ${ }^{1}$ Department of Cardiology and Cardiovascular Interventions, University Hospital, Krakow, Poland \\ ${ }^{2}$ Department of Intensive Care and Perioperative Medicine, Jagiellonian University Medical College, Krakow, Poland \\ ${ }^{3} 2^{\text {nd }}$ Department of Cardiology, Institute of Cardiology, Jagiellonian University Medical College, Krakow, Poland
}

Adv Interv Cardiol 2021; 17, 2 (64): 155-162

DOI: https://doi.org/10.5114/aic.2021.107493

\begin{abstract}
A bstract
There is a worldwide expansion in percutaneous therapy for valvular heart disease. Rapidly evolving technology and the general increase in life expectancy will support the evolution of new treatment options dedicated to structural heart interventions. Transcatheter aortic valve implantation for severe aortic valve stenosis and percutaneous mitral valve repair with the MitraClip system for severe mitral regurgitation have been demonstrated as a feasible, innovative alternative for surgical treatment. Despite the inequality in clinical experience, both procedures have encouraging results and now are a part of everyday clinical practice. More importantly, rapid development is expected in the next decades. However, the global coronavirus disease 2019 (COVID-19) pandemic imposed redistribution of healthcare resources. Hospitals were obliged to modify their workflow and limit TAVI and MitraClip procedures to urgent or in highly symptomatic patients. Despite this encumbrance improvement in technology and experience supported by robust evidence from current studies might extend indications for both procedures. The future holds promise for this treatment modality to become the preferred procedure for all patients despite age or risk and reserving surgical treatment for a minority. Thus, we present state-of-the-art and current evidence for both methods assumed to change the paradigm of treatment of valvular heart failure in the future.
\end{abstract}

Key words: transcatheter aortic valve replacement, valvular heart disease, primary mitral regurgitation, secondary mitral regurgitation, heart failure.

\section{Introduction}

There is a worldwide expansion in percutaneous therapy for valvular heart disease [1-6]. Rapidly evolving technology and the general increase in life expectancy will support the evolution of new treatment options dedicated to structural heart interventions. Transcatheter aortic valve implantation (TAVI) for severe aortic valve stenosis (AS) and percutaneous mitral valve repair with the MitraClip system for severe mitral regurgitation (MR) have been demonstrated as a feasible, innovative alternative for surgical treatment [1-6]. The first TAVI procedure was performed in 2002, and just 1 year later early, experience with MitraClip was reported [7, 8]. However, the complexity and diversity of the mitral valve and subvalvular apparatus have resulted in slower development of percutaneous techniques dedicated to the mitral valve as compared to TAVI. Furthermore, an exponentially increasing number of TAVI procedures was related to the ageing population across Europe. It might also be a result of the Valve for Life initiative implemented by the European Association of Percutaneous Coronary Interventions (EAPCI) [9-11]. Despite the inequality in clinical experience [3, 9-11], both procedures have encouraging results and now are a part of everyday clinical practice. More importantly, prompt development is expected in the next decades.

Thus, we present state-of-the-art and current evidence for both methods assumed to change the paradigm of treatment of valvular heart failure (HF) in the future.

\section{MitraClip}

Mitral regurgitation (MR) is the second most frequent valvular heart disease, with a prevalence of up to $10 \%$ 
in the older population [12]. Despite the detrimental effect on prognosis, approximately half of the patients with severe $M R$ are not eligible for surgery due to an unacceptably high perioperative risk [12]. According to the current European Society of Cardiology (ESC) guidelines, MR is divided into primary (organic, degenerative) and secondary (functional, ischemic) [13]. Defects in the morphology of the mitral valve apparatus lead to primary $M R$. In the secondary $M R$, coaptation is impaired by left ventricular (LV) remodeling and annular dilatation or papillary muscle displacement. Thus, it is related to LV dysfunction rather than the mitral valve itself. However, both types are associated with an unfavorable prognosis without invasive treatment [13]. Surgical correction is the treatment of choice. However, the abundance of inoperable patients caused an emerging need for percutaneous technique restoring coaptation of the mitral valve [13].

The MitraClip (Abbott Laboratories, Menlo Park, California, USA) is a 4-mm cobalt-chromium clip covered with a polypropylene fabric. The technique aims to mimic the surgical procedures known as edge-to-edge repair or "Alfieri's stitch" [14]. Free edges of the anterior and posterior valvular leaflet are linked by a clip and create a "double-orifice" mitral valve. The procedure provides a significant reduction of MR severity with a favorable safety profile. Implantation is conducted via the femoral vein under general anesthesia with fluoroscopy and transesophageal echocardiography navigation. The device may be reopened with repeated attempts of implantation. In the case of a suboptimal result, a second or even third clip may be delivered. The MitraClip system received initial CE-Mark approval in Europe in 2008 and was approved by the Food and Drug Administration (FDA) in 2013 for use in primary MR. Furthermore, in 2019, it was endorsed for use in functional MR in patients at prohibitive risk for mitral valve surgery after evaluation by an interdisciplinary heart team. However, the 2017 update of the American Heart Association/American College of Cardiology valve guidelines suggested the use of MitraClip in severe primary MR among highly symptomatic patients (New York Heart Association class III to IV) despite optimal medical therapy, with favorable anatomy, reasonable life expectancy and high surgical risk due to comorbidities. Patients with primary MR should be evaluated by the heart team for feasibility and potential risk and benefit from the procedure [15]. In contrast, the 2017 ESC guidelines allow the percutaneous edge-to-edge procedure to be considered in patients with symptomatic severe secondary MR with suitable valve morphology, when revascularization is not indicated and the procedural risk is low. Most of these recommendations were driven by the data from Endovascular Valve Edge to Edge Repair Study (EVEREST) I and II. Based on findings in animal models, EVEREST I was lunched as a phase I safety and feasibility trial. The study group consisted of 27 patients with at least moder- ate-to-severe MR $(\geq 3+)$. The device was successfully implanted in 22 patients, while 3 patients underwent the percutaneous procedure with no clip due to inadequate reduction of MR. All of these 3 patients underwent subsequent surgical treatment. Furthermore, two other patients were referred to surgery due to device malfunctions. Among 22 patients with an implanted MitraClip, a significant reduction of MR at 1-month follow-up was observed in 14 cases. Majority of them maintained this improvement at 6 months. Freedom from surgical treatment was reported in $82 \%(18 / 22)$ after 6 months. No major adverse events were observed in $85 \%$ at 1 month. One patient suffered from a stroke, and 3 patients experienced clip replacement from one leaflet. The study protocol was modified at the beginning to allow implantation of multiple clips [16]. A follow-up study analyzed 107 patients (including the original cohort from EVEREST I) with moderate-to-severe $(3+)$ or severe $(4+)$ both organic (79\%) and functional (21\%) MR. Procedural success defined by the investigators as MR less than 2+ was achieved in $74 \%$ of patients undergoing MitraClip placement with a comparable outcome in degenerative and functional MR. At 1-year follow-up, $66 \%$ remained free of death, MR greater than $2+$ or mitral surgery. No periprocedural deaths were reported, and 10 (9.3\%) patients experienced an adverse event at 1-month follow-up. This initial clinical experience with MitraClip device made it possible to establish the feasibility and safety of the procedure [6]. In 2011, EVEREST II was initiated as the first randomized clinical trial comparing the efficacy and safety of surgical treatment with MitraClip [17]. Patients were randomized $2: 1$ to percutaneous therapy versus surgery. The study group included 258 patients with MR $3+$ or $4+(27 \%$ secondary and $73 \%$ primary). The use of MitraClip was associated with a lower rate of periprocedural complications as compared to surgery. However, efficacy in the decrease of MR severity was lower for the percutaneous treatment. Importantly, patients with improvement in MR grade after MitraClip maintained these results during 24 months of follow-up. Furthermore, no differences in mortality were observed between groups. Based on subgroup analysis, the most beneficial outcome in the MitraClip group was observed in older patients ( $\geq 70$ years), in functional MR and low LV ejection fraction [17]. Several subsequent studies confirmed the EVEREST I and II outcomes. The Amsterdam Center for Contemporary European Studies in Europe (ACCESS-EU) showing data of "real-world" experience enrolled 567 patients with significant MR (77\% functional MR). At 12-month follow-up, an $81.8 \%$ survival rate with MR grade $2+$ or less maintained in $78.9 \%$ of patients was reported. Furthermore, functional improvement measured in NYHA scale and distance in the 6-minute walk test as well as amelioration of quality of life (QoL) was observed up to 12 months after the MitraClip procedure. In addition, MitraClip was associated with low rates of 
hospital mortality and adverse events [18]. Getting Reduction of Mitral Insufficiency by Percutaneous Clip Implantation (GRASP) was another registry with promising results. At 12 months, the composite event-free survival endpoint of death, surgery, or $M R \geq 3+$ was $75.8 \%$ for 117 patients treated with MitraClip. Furthermore, there were no differences in safety or efficacy outcomes for both degenerative and functional MR [19]. A meta-analysis of 16 studies reported similar results with a low adverse event profile with $14.7 \%$ of patients with $M R \geq 3+$ during 12-month follow-up [20]. Furthermore, data from the largest registry to date, from Germany, demonstrated $79.7 \%$ survival among all MitraClip patients at 12 months as well as a decreased rate of hospital readmissions due to HF. However, this study did not provide echocardiographic data at 12 months. Outcomes available in 828 patients showed clinical benefit with $63 \%$ of patients in NYHA I or II class as compared with $11 \%$ at baseline. The majority of patients were independent in self-care after MitraClip [21]. Finally, recently, two randomized controlled trials brought new light to our knowledge, despite apparently contradictory information [22, 23]. The Cardiovascular Outcomes Assessment of the MitraClip Percutaneous Therapy (COAPT) trial was designed to determine the safety and effectiveness of the MitraClip for functional MR in patients with HF as compared to standard-ofcare therapy [23]. Patients disqualified from mitral valve surgery after heart team assessment were enrolled in the study. Percutaneous Repair with the MitraClip Device for Severe Functional/Secondary Mitral Regurgitation (MITRA-FR) evaluated the benefits and safety of the MitraClip system with optimal medical therapy (OMT) as compared with OMT alone in severe symptomatic secondary MR with contraindication to surgical repair [22]. The primary endpoint of both trials was based on clinical outcomes. Death or readmission to hospital related to exacerbation of HF at 12 months in MITRA-FR, and all hospitalizations due to HF at 24 months in COAPT. No differences between the two arms in MITRA-FR were observed, while the MitraClip decreased mortality $(29.1 \%$ vs. $46.1 \%$; $p=0.001)$ and hospitalizations related to $\mathrm{HF}(35.8 \%$ vs. $67.9 \% ; p=0.001$ ) and improved QoL and functional capacity in the COAPT trial. The absolute risk reduction of mortality in the COAPT trial was $17 \%$, with a number needed to treat of 6 for prevention of one death over 24 months. Importantly, such an impressive effect in reduction of mortality was previously reported for the use of renin-angiotensin inhibitors in HF. Importantly, an echocardiographic substudy of the COAPT Trial confirmed that benefits of MitraClip were observed in all echocardiographic subgroups, regardless of the degree of LV dysfunction, LV dimensions, pulmonary hypertension, the severity of tricuspid regurgitation or individual mitral regurgitation parameters. Also, the QoL substudy confirmed that in patients with HF and secondary mitral regurgita- tion, MitraClip implantation resulted in early, substantial and sustained improvement in health status compared with standard care [24]. Thus, these results might lead to a paradigm shift in the treatment of patients with mitral valve regurgitation and HF. However, the outstanding clinical effect and inconsistent results of these two trials encourage discussion on a potential explanation. Despite the approval by the FDA for MitraClip in secondary MR, these data should be viewed with caution. First, the sample size was much larger in the COAPT trial as compared to the MITRA-FR. Furthermore, patients enrolled in the MITRA-FR trial had larger LV end-diastolic volumes (MITRA-FR vs. COAPT: $135 \pm 35 \mathrm{ml} / \mathrm{m}^{2}$ vs. $101 \pm 34 \mathrm{ml} / \mathrm{m}^{2}$, respectively), suggesting more advanced LV dysfunction. Also, the COAPT trial excluded patients with extremely dilated LV (end-systolic diameter $<70 \mathrm{~mm}$ ), while MITRA-FR had no LV dimension limit. Patients with HF and concomitant MR and severe LV dilation were associated with worse outcomes after surgical correction of ischemic MR [25]. In addition, there was a difference in LV ejection fraction inclusion criteria (MITRA-FR vs. COAPT: $15-40 \%$ vs. 20-50\%, respectively). However, the mean LV ejection fraction in the MitraClip arm was comparable (MITRA-HF vs. COAPT: $33.3 \%$ vs. $31.3 \%$ ). In addition, there was inconsistency in the severity of MR in both trials (MITRA-FR vs. COAPT effective regurgitant orifice area (EROA): $31 \pm 10$ $\mathrm{mm}^{2}$ vs. $41 \pm 15 \mathrm{~mm}^{2}$ ). In the MITRA-FR trial, 2012 European guidelines criteria were applied, while the COAPT trial was based on the 2006/2008 American guidelines. More clips were implanted per patient in the COAPT trial as compared to MITRA-FR. Thus, a greater reduction of MR was observed in the COAPT trial. Also, over $30 \%$ of patients in both groups in the COAPT trial were not included in the 24-month visits, which might limit the results. These factors may explain the striking discrepancies in outcomes between the MITRA-FR and COAPT trials [26, 27]. However, the observed outcomes should be extrapolated with caution and only carefully selected patients, evaluated by a multidisciplinary heart team, should be referred for the MitraClip procedure for the treatment of secondary MR. We should keep in mind that patients scheduled for the MitraClip should meet strict echocardiographic criteria. Hopefully, the introduction of new devices, for instance, Cardioband and Harpoon, may address other populations of patients with mitral valve disease. Results of the Reshape HF2 trial will bring more data and hopefully will strengthen the role of the MitraClip in the treatment of secondary MR [26-28]. Furthermore, randomized trials with longer-term observation should be performed to establish the role of the MitraClip.

\section{Transcatheter aortic valve implantation}

Severe AS is the most common valvular disease. The incidence tends to increase rapidly with age to $2.5-8.1 \%$ in the population over 75 years old [29-32]. The progno- 
sis in symptomatic severe AS is poor; average survival time is 2-3 years [29-32]. Three processes are involved in its etiology: lipid accumulation, inflammation, and calcification [1-3]. Several mechanisms and risk factors involved in the pathogenesis of AS are similar to those observed in atherosclerosis. Reduced valve area leads to progressively increased left ventricular pressure overload and left ventricular hypertrophy as a major compensatory mechanism. Finally, it leads to reduced ventricular ejection performance and cardiac output with HF syndrome as a consequence. The observed and forecasted changes in the demographic structure of the population indicate significant advances in the population aging process. It will result in an exponential increase in the need for AS treatment in the coming years. Currently, the treatment of choice of symptomatic severe AS is still surgical aortic valve replacement (SAVR). However, one-third of elderly patients are not even qualified for surgery due to age, comorbidities, and too high risk of surgery. Since the first pioneering procedure by Cribier et al. in 2002, TAVI has become an acceptable standard of care in patients with symptomatic severe AS who are not eligible for surgery. After several first-in-man trials, Edwards Sapien Valve and CoreValve received the CE mark in 2007 as the first TAVI prostheses on the European market [33-35]. The Placement of AoRTic TraNscathetER Valve (PARTNER) 1 trial demonstrated the superiority of TAVI with the Edwards Sapien valve system (Edwards Lifesciences, Irvine, CA, USA) over optimal medical treatment. Thus, TAVI has been considered as a feasible alternative for inoperable high-risk patients [1]. Beneficial outcomes with the self-expandable CoreValve system (CoreValve, Irvine, CA, USA) were also demonstrated by the CoreValve US Pivotal Trial [36]. Furthermore, studies with 5-year outcomes of TAVI and national registries provided additional evidence supporting favorable results of novel treatment option [37-40]. However, in both trials, major vascular complications were more frequently reported in TAVI, while higher rates of major bleeding and new-onset atrial fibrillation (AF) were observed after SAVR. Rapidly evolving technology allowed the indications for TAVI to be expanded to intermediate- and lowrisk patients. Comparable outcomes in terms of both allcause mortality and disabling stroke for TAVI and SAVR were reported in the PARTNER 2 cohort A study at 2 years after the procedure [2]. Furthermore, in the transfemoral cohort, TAVI even resulted in a lower rate of adverse events than SAVR (HR $=0.79 ; 95 \% \mathrm{Cl}$ : 0.62-1.00). In addition, TAVI resulted in lower 30-day risk of acute kidney injury (AKI), severe bleeding and new-onset AF. In contrast, lower rates of moderate or severe paravalvular aortic regurgitation (PVR) and major vascular complications were reported in SAVR. Importantly, patients in the TAVI group with moderate or severe PVR at 30 days were associated with higher mortality during 24-month fol- low-up as compared to the group with no or trace PVR. Surgical or Transcatheter Aortic-Valve Replacement in Intermediate-Risk Patients (SURTAVI) also demonstrated non-inferiority of TAVI as compared to SAVR in patients with severe AS at intermediate risk [41]. The incidence of the composite endpoint of all-cause death or disabling stroke at 24 months was similar in both groups. Patients after surgical treatment experienced higher 30-day rates of $\mathrm{AKI}, \mathrm{AF}$ and blood transfusion. On the other hand, a higher rate of major vascular complications and need for permanent pacemaker implantation were observed for TAVI. Moderate or severe PVR was more common in the TAVI group in 12-month observation as compared to the SAVR group. Furthermore, Nordic Aortic Valve Intervention Trial (NOTION) investigated relatively low risk patients with comparable rates of all-cause mortality, stroke, or myocardial infarction at 12 months (TAVI vs. SAVR, $13.1 \%$ Vs. $16.3 \% ; p=0.43$ ) and at 5 years (TAVI vs. SAVR, $39.2 \%$ vs. $38.5 \% ; p=0.78$ ) [41]. Finally, a meta-analysis of randomized controlled trials suggested lower risk of death in all TAVI patients despite the risk profile (HR $=0.87 ; 95 \% \mathrm{Cl}: 0.76-0.99 ; p=0.038)$ [42] and in TAVI with transfemoral access in both intermediate and low risk ( $\mathrm{HR}=0.79 ; 95 \% \mathrm{Cl}: 0.66-0.94)$ [43]. Another meta-analysis of 5647 intermediate-risk patients suggested no differences in all-cause and cardiac mortality at 30-day, 12-month and 24-month follow-up. Patients treated with SAVR were at higher risk of AKI and AF. Need for permanent pacemaker implantation and aortic insufficiency were more frequently reported in patients treated with TAVI [44]. Recent data on 20,549 patients with severe AS and low surgical risk from the German Aortic Valve Registry (GARY) demonstrated that TAVI was associated with improved 30-day survival as compared to SAVR (98.3\% vs. 97.0\%; $p=0.001)$ [45]. Similar outcomes were reported in both groups in 12-month observation (90.4\% vs. $91.2 \% ; p=0.40)$. Age was the most relevant factor influencing decisions of choice between TAVI and SAVR (mean age: 78.9 vs. 67.5 years, respectively). Due to the above-mentioned encouraging results, TAVI is on the way to becoming a universal treatment even in low-risk cohorts. Finally, data from PARTNER 3 and Evolut Low Risk trials allowed the FDA to simultaneously expand the indications for the use of the Edwards Lifesciences' Sapien 3 valve and Medtronic's CoreValve Evolut system to low-risk patients with severe AS [3, 46]. The PARTNER 3 Trial demonstrated that low-risk patients treated with TAVI experienced lower incidence of the primary composite endpoint (death, stroke, or rehospitalization at 1 year) than the SAVR group (8.5\% vs. $15.1 \%$; absolute difference, -6.6 percentage points; $95 \% \mathrm{Cl}:-10.8$ to -2.5$)$. The results were valid for both noninferiority and superiority criteria. At 30 days, lower rates of stroke and new-onset AF were observed in the TAVI group. There were no differences in the rate of mortality, permanent pacemaker in- 
sertions and moderate to severe PVR between groups. Furthermore, these outcomes were maintained up to 12 months [3]. Similarly, the Evolut Low Risk trial demonstrated no differences between TAVI and SAVR in the incidence of the primary composite endpoint (death or disabling stroke) at 24 months for the noninferiority threshold. At 30 days, TAVI patients were less likely to have undergone disabling stroke, bleeding complications, AKI or AF. However, moderate or severe PVR and permanent pacemaker implantation were more common in the TAVI population as compared with SAVR. Similar mortality rates were reported in both study arms at 30 days and 12 months. Furthermore, admission to hospital for HF was less frequent in the TAVI group at 12-month follow-up [46]. There were substantial differences in the design of both studies, populations as well as composite endpoints. In the PARTNER 3 trial, more patients were excluded due to severe calcifications of the left ventricular outflow tract, adverse aortic root, and burdensome transfemoral access. It might partly explain the low rates of TAVI complications observed in this trial. In contrast to the PARTNER 3 trial, rehospitalizations at 12 months were not included in the composite endpoint of the Evolut Low Risk trial. However, a reduction in hospitalizations for HF at 12 months in the Evolut Low Risk trial may suggest that the performance of self-expanding valves and balloon-expandable valves is likely to be similar in this term [46]. Despite inconclusive outcomes of meta-analyses [47-50], available data suggest at least noninferiority of TAVI as compared to surgical treatment. Thus, TAVI could be considered as a first-line therapy in low risk patients with severe aortic stenosis. However, long-term observation is pivotal to validate these outcomes.

\section{Future and perspectives}

Nowadays, approximately 180000 patients might be considered as potential TAVI candidates in the European Union and in Northern America each year. This population will probably increase to 270000 after expanded indications for low-risk patients [51]. The astounding improvement in technology and experience supported by robust evidence from current studies will lead to inevitable development and domination of the TAVI procedure in the treatment of severe AS. Implementation of TAVI in facilities without on-site cardiac surgery is another crucial step to increase availability of this procedure. Furthermore, the next milestone will be the growing need for valve-invalve procedures and paravalvular leak closures [52-54]. Adoption of these techniques might allow interventional cardiology to effectively deal with complications of both surgical and transcatheter valve interventions. Another important issue is device-host interactions and the difficulty to predict optimal suitability of the valve in the wide variability of geometry and dimensions between particular patients $[52,53,55]$. The growing number of TAVI candidates and available valve types will require a patient-tailored approach and appropriate tools for procedural planning [52-57]. Another milestone for TAVI development is bicuspid aortic valve, as the most common congenital heart disease [52-57]. There is a lack of data comparing TAVI and SAVR in this specific anatomical setting. However, promising results with low complication rates were reported recently [52-57]. Longevity of the TAVI valve will also be an important issue to solve. Facing expansion of this device to a younger population will necessitate evaluation and development of durability of the biological prosthesis $[52,53]$. Since TAVI is considered in a younger, low-risk population, risk of future interventions such as percutaneous coronary intervention will be increased. Thus, position of the aortic valve prosthesis might be an important challenge for access to coronary arteries $[52,53]$. Also proper risk stratification for decision-making and TAVI qualification are required [52-54]. Patient selection, device type and access, with comprehensive evaluation based on a patient's medical condition, will be key for TAVI utilization $[52-54,58]$. The future holds promise for this treatment modality to become the preferred procedure for all patients despite age or risk and reserving surgical treatment for a minority of cases.

\section{TAVI and MitraClip - Polish experience}

A team of invasive cardiologists from the University Hospital and cardiac surgeons from the John Paul II Specialist Hospital in Krakow performed the first TAVI procedure in Poland in 2008 and MitraClip 5 years later. Despite rapid implementation of both techniques to daily clinical practice, the number of MitraClip procedures in Poland remained unsatisfactory $[9,10,59]$. However, the total number increases each year [59]. New indications for MitraClip might allow a new population of patients to benefit from this device. Similarly, despite continuous growth of the number of TAVI, with 40 procedures per million population in 2019, it is still far below the average volume in most Western European countries [9, 10, 59]. Several explanations for this disparity are possible, including low national expenses on health care, and the relatively high cost of the procedure after adjustment for gross domestic product per capita. Also, the Polish National Health Fund strategy of restrictive reimbursement of the TAVI resulted in a limited annual frequency of this procedure. Thus, waiting lists for TAVI exceed current possibilities provided by the national healthcare system. All these factors together with aging of the population entailed an unmet need for enhancement of TAVI volume in Poland. On the basis of these alarming reports, the EAPCI introduced the Valve for Life Initiative $[11,60]$. The main aim was to enhance awareness of inequality of patient access to this life-saving therapy and increase the number 
of TAVI procedures per year. Furthermore, the initiative also aimed to create a suitable climate for serious discussion about enhancing reimbursement for TAVI. As a result of a well-planned and focused nationwide campaign, the number of patients treated with TAVI is increasing every year. However, extending indications to low-risk patients will exponentially increase the list of candidates waiting for less invasive treatment $[9,10,59]$. These findings are essential for health care resource planning of structural heart disease treatment. Thus, constant development and support of this technique are crucial for the national healthcare system and aging population.

\section{Structural procedures during the COVID-19 pandemic}

The global coronavirus disease 2019 (COVID-19) pandemic necessitated redistribution of healthcare resources for treatment of patients infected with the severe acute respiratory syndrome coronavirus 2 (SARS-CoV-2). Thus, hospitals were obliged to modify their workflow, especially the number of elective procedures using valuable ventilators and beds in intensive care units. Many hospitals decided to limit TAVI and MitraClip procedures to urgent cases or highly symptomatic patients [61]. Recently, a few position papers postulated recommendations for percutaneous treatment of structural heart diseases during the COVID-19 pandemic [61-64]. Limited anesthetic and intensive care facilities raise the need for triage of patients and performing procedures only in selected cases with minimal resources. Furthermore, sameor next-day discharge is desirable to avoid potential risks of COVID-19 transmission and to provide cardiovascular care to high-risk patients. With pandemic abatement, healthcare systems might face substantial waiting lists and a high burden of patients with progression of symptoms. Significant delay in treatment might be associated with detrimental outcomes even in populations initially considered as low risk [61-64].

\section{Conflict of interest}

The authors declare no conflict of interest.

\section{References}

1. Leon MB, Smith CR, Mack M, et al. Transcatheter aortic-valve implantation for aortic stenosis in patients who cannot undergo surgery. PARTNER Trial Investigators. N Engl J Med 2010; 363 : 1597-607.

2. Leon MB, Smith CR, Mack MJ, et al. Transcatheter or surgical aortic-valve replacement in intermediate-risk patients. N Engl J Med 2016; 374: 1609-20.

3. Mack MJ, Leon MB, Thourani VH, et al.; PARTNER 3 Investigators. Transcatheter Aortic-Valve Replacement with a Balloon-Expandable Valve in Low-Risk Patients. N Engl J Med 2019; 380: 1695-705.

4. Feldman T, Foster E, Glower DD, et al.; EVEREST II Investigators. Percutaneous repair or surgery for mitral regurgitation. N Engl J Med 2011; 364: 1395-406.
5. Glower DD, Kar S, Trento A, et al. Percutaneous mitral valve repair for mitral regurgitation in high-risk patients: results of the EVEREST II study. J Am Coll Cardiol 2014; 64: 172-81.

6. Feldman T, Kar S, Rinaldi $M$, et al.; EVEREST Investigators. Percutaneous mitral repair with the MitraClip system: safety and midterm durability in the initial EVEREST (Endovascular Valve Edge-to-Edge REpair Study) cohort. J Am Coll Cardiol 2009; 54: 686-94.

7. Cribier A, Eltchaninoff $\mathrm{H}$, Bash A, et al. Percutaneous transcatheter implantation of an aortic valve prosthesis for calcific aortic stenosis: first human case description. Circulation 2002; 106: 3006-8.

8. Condado JA, Acquatella H, Rodriguez L, et al. Percutaneous edgeto-edge mitral valve repair: 2-year follow-up in the first human case. Catheter Cardiovasc Interv 2006; 67: 323-5.

9. Legutko J, Siudak Z, Parma R, et al. Poland: coronary and structural heart interventions from 2010 to 2015 . Eurolntervention 2017; 13: Z51-4.

10. Dudek D, Barbato E, Baumbach A, et al. Current trends in structural heart interventions: an overview of the EAPCI registries. Eurolntervention 2017; 13: Z11-3.

11. Windecker S, Haude M, Baumbach A. Introducing a new EAPCI programme: the valve for life initiative. Eurolntervention 2016; 11: 977-9.

12. Van Mieghem N, Piazza N, Anderson RH, et al. Anatomy of the mitral valvular complex and its implications for transcatheter interventions for mitral regurgitation. J Am Coll Cardiol 2010; 56: 617-26.

13. Baumgartner H, Falk V, Bax JJ, et al.; ESC Scientific Document Group. 2017 ESC/EACTS Guidelines for the management of valvular heart disease. Eur Heart J 2017; 38: 2739-91.

14. Alfieri O, Maisano F. An effective technique to correct anterior mitral leaflet prolapse. J Card Surg 1999; 14: 468-70.

15. Nishimura RA, Otto CM, Bonow RO, et al. 2017 AHA/ACC Focused Update of the 2014 AHA/ACC Guideline for the Management of Patients With Valvular Heart Disease: A Report of the American College of Cardiology/American Heart Association Task Force on Clinical Practice Guidelines. Circulation 2017; 135: 1159-95.

16. Feldman T, Wasserman HS, Herrmann HC, et al. Percutaneous mitral valve repair using the edge-to-edge technique: six-month results of the EVEREST phase i clinical trial. J Am Coll Cardiol 2005; 46: 2134-40.

17. Feldman T, Foster E, Glower DD, et al. Percutaneous repair or surgery for mitral regurgitation. N Engl J Med 2011; 364: 1395-406.

18. Maisano F, Franzen O, Baldus S, et al. Percutaneous mitral valve interventions in the real world: early and 1-year results from the ACCESS-EU, a prospective, multicenter, nonrandomized post-approval study of the MitraClip therapy in Europe. J Am Coll Cardiol 2013; 62: 1052-61.

19. Grasso C, Capodanno D, Scandura S, et al. One- and twelvemonth safety and efficacy outcomes of patients undergoing edge-to-edge percutaneous mitral valve repair (from the GRASP Registry). Am J Cardiol 2013; 111: 1482-7.

20. Vakil K, Roukoz H, Sarraf M, et al. Safety and efficacy of the MitraClip(R) system for severe mitral regurgitation: a systematic review. Catheter Cardiovasc Interv 2014; 84: 129-36.

21. Puls M, Lubos E, Boekstegers P, et al. One-year outcomes and predictors of mortality after MitraClip therapy in contemporary clinical practice: results from the German transcatheter mitral valve interventions registry. Eur Heart J 2016; 37: 703-12. 
22. Obadia JF, Messika-Zeitoun D, Leurent G, et al.; MITRA-FR Investigators. Percutaneous repair or medical treatment for secondary mitral regurgitation. N Engl J Med 2018; 379: 2297-306.

23. Stone GW, Lindenfeld J, Abraham WT, et al.; COAPT Investigators. Transcatheter mitral-valve repair in patients with heart failure. N Engl J Med 2018; 379: 2307-18.

24. Arnold SV, Chinnakondepalli KM, Spertus JA, et al.; COAPT Investigators. Health status after transcatheter mitral-valve repair in heart failure and secondary mitral regurgitation: COAPT trial. J Am Coll Cardiol 2019; 73: 2123-32.

25. Braun J, van de Veire NR, Klautz RJ, et al. Restrictive mitral annuloplasty cures ischemic mitral regurgitation and heart failure. Ann Thorac Surg 2008; 85: 430-6.

26. Garima A, Nirav P, Pankaj A. Futile MITRA-FR and a positive COAPT trial: where does the evidence leave the clinicians? Int J Cardiol Heart Vasc 2019; 22: 18-9.

27. Pibarot P, Delgado V, Bax J. MITRA-FR vs. COAPT: lessons from two trials with diametrically opposed results. Eur Heart J Cardiovasc Imaging 2019; 20: 620-4.

28. A Clinical Evaluation of the Safety and Effectiveness of the MitraClip System in the Treatment of Clinically Significant Functional Mitral Regurgitation (Reshape-HF2). ClinicalTrials.gov Website. https://clinicaltrials.gov/ct2/show/NCT02444338

29. Tokarek T, Sobczyński R, Dziewierz A, et al. Clinical outcomes in patients after surgical and transcatheter aortic valve replacement. Pol Arch Med Wewn 2015; 125: 755-64.

30. Tokarek T, Siudak Z, Dziewierz A, et al. Assessment of quality of life in patients after surgical and transcatheter aortic valve replacement. Catheter Cardiovasc Interv 2016; 88: E80-8.

31. Dziewierz A, Tokarek T, Kleczynski P, et al. Impact of chronic obstructive pulmonary disease and frailty on long-term outcomes and quality of life after transcatheter aortic valve implantation. Aging Clin Exp Res 2018; 30: 1033-40.

32. Olszewska K, Tokarek T, Bętkowska-Korpała B, et al. Assessment of cognitive functions and quality of life in patients scheduled for transcatheter aortic valve implantation: a pilot study. Adv Interv Cardiol 2017; 13: 258-62.

33. Reardon MJ, Van Mieghem NM, Popma JJ, et al. Surgical or transcatheter aortic-valve replacement in intermediate-risk patients. N Engl J Med 2017; 376: 1321-31.

34. Cribier A, Eltchaninoff $\mathrm{H}$, Tron C, et al. Treatment of calcific aortic stenosis with the percutaneous heart valve: mid-term follow-up fromthe initial feasibility studies: the French experience. J Am Coll Cardiol 2006; 47: 1214-23.

35. Webb JG, Pasupati S, Humphries K, et al. Percutaneous transarterial aortic valve replacement in selected high risk patients with aortic stenosis. Circulation 2007; 116: 755-63.

36. Adams DH, Popma JJ, Reardon MJ, et al.; U.S. CoreValve Clinical Investigators. Transcatheter aortic-valve replacement with a self-expanding prosthesis. N Engl J Med 2014; 370: 1790-8.

37. Gleason TG, Reardon MJ, Popma JJ, et al.; CoreValve U.S. Pivotal High Risk Trial Clinical Investigators. 5-year outcomes of self-expanding transcatheter versus surgical aortic valve replacement in high-risk patients. J Am Coll Cardiol 2018; 72: 2687-96.

38. Mack MJ, Leon MB, Smith CR, et al. 5-year outcomes of transcatheter aortic valve replacement or surgical aortic valve replacement for high surgical risk patients with aortic stenosis (PARTNER 1): a randomised controlled trial. Lancet 2015; 385: 2477-84.

39. Hamm CW, Möllmann H, Holzhey D, et al.; GARY-Executive Board. The German Aortic Valve Registry (GARY): in-hospital outcome. Eur Heart J 2014; 35: 1588-98.
40. Gilard M, Eltchaninoff H, Donzeau-Gouge P, et al.; FRANCE 2 Investigators. Late outcomes of transcatheter aortic valve replacement in high-risk patients: The FRANCE-2 Registry. J Am Coll Cardiol 2016; 68: 1637-47.

41. Reardon MJ, Van Mieghem NM, Popma JJ, et al. Surgical or transcatheter aortic-valve replacement in intermediate-risk patients. N Engl J Med 2017; 376: 1321-31.

42. Siontis GC, Praz F, Pilgrim T, et al. Transcatheter aortic valve implantation vs. surgical aortic valve replacement for treatment of severe aortic stenosis: a meta-analysis of randomized trials. Eur Heart J 2016; 37: 3503-12.

43. Siemieniuk RA, Agoritsas T, Manja V, et al. Transcatheter versus surgical aortic valve replacement in patients with severe aortic stenosis at low and intermediate risk: systematic review and meta-analysis. BMJ 2016; 354: i5130.

44. Lazkani M, Singh N, Howe C, et al. An updated meta-analysis of TAVR in patients at intermediate risk for SAVR. Cardiovasc Revasc Med 2019; 20: 57-69.

45. Bekeredjian R, Szabo G, Balaban Ü, et al. Patients at low surgical risk as defined by the Society of Thoracic Surgeons Score undergoing isolated interventional or surgical aortic valve implantation: in-hospital data and 1-year results from the German Aortic Valve Registry (GARY). Eur Heart J 2019; 40: 1323-30.

46. Popma JJ, Deeb GM, Yakubov SJ, et al.; Evolut Low Risk Trial Investigators. Transcatheter aortic-valve replacement with a self-expanding valve in low-risk patients. N Engl J Med 2019; 380: 1706-15.

47. Levett JY, Windle SB, Filion KB, et al. Meta-analysis of transcatheter versus surgical aortic valve replacement in low surgical risk patients. Am J Cardiol 2020; 125: 1230-8.

48. Kolkailah AA, Doukky R, Pelletier MP, et al. Transcatheter aortic valve implantation versus surgical aortic valve replacement for severe aortic stenosis in people with low surgical risk. Cochrane Database Syst Rev 2019; 12: CD013319.

49. Witberg G, Landes U, Lador A, et al. Meta-analysis of transcatheter aortic valve implantation versus surgical aortic valve replacement in patients at low surgical risk. Eurolntervention 2019; 15: e1047-56.

50. Rawasia WF, Usman MS, Mujeeb FA, et al. Transcatheter versus surgical aortic valve replacement in low-surgical-risk patients: a meta-analysis of randomized-controlled trials and propensity-matched studies. Cardiovasc Revasc Med 2020; 21: 612-8.

51. Durko AP, Osnabrugge RL, Van Mieghem NM, et al. Annual number of candidates for transcatheter aortic valve implantation per country: current estimates and future projections. Eur Heart J 2018; 39: 2635-42.

52. Barbanti M, Webb JG, Dvir D, et al. Residual challenges in TAVI: moving forward. Eurolntervention 2019; 15: 857-66.

53. Howard C, Jullian L, Joshi M, et al. TAVI and the future of aortic valve replacement. J Card Surg 2019; 34: 1577-90.

54. Vrachatis DA, Vavuranakis M, Tsoukala S, et al. TAVI: valve in valve. A new field for structuralists? Literature review. Hellenic J Cardiol 2020; 61: 148-53.

55. Bompotis G, Meletidou M, Karakanas A, et al. Transcatheter aortic valve implantation using 3-D printing modeling assistance. A single-center experience. Hellenic J Cardiol 2020; 61: 131-2.

56. Bhardwaj B, Golwala H, Balla S, et al. Transcatheter aortic valve replacement in patients with bicuspid aortic valve: insights from the National Inpatient Sample database. Hellenic J Cardiol 2019; 60: 394-5. 
57. Makkar RR, Yoon SH, Leon MB, et al. Association between transcatheter aortic valve replacement for bicuspid vs tricuspid aortic stenosis and mortality or stroke. JAMA 2019; 321: 2193-202.

58. Ha FJ, Bissland K, Mandrawa C, et al. Frailty in patients with aortic stenosis awaiting intervention: a comprehensive review. Intern Med J 2021; 51: 319-26.

59. Dudek D, Siudak Z, Grygier M, et al. Interventional cardiology in Poland in 2019. Summary report of the Association of Cardiovascular Interventions of the Polish Cardiac Society (AISN PTK) and Jagiellonian University Medical College. Adv Interv Cardiol 2020; 16: 123-6.

60. Witkowski A, Gilard M. EAPCI Focus on the EAPCI Valve for Life initiative update. Eurolntervention 2016; 12: 938.

61. Wood DA, Sathananthan J. "Minimalist" transcatheter aortic valve implantation during the COVID-19 pandemic: previously optional but now a necessity. Eurolntervention 2020; 16: e451-2.

62. Shah PB, Welt FGP, Mahmud E, et al. Triage considerations for patients referred for structural heart disease intervention during the coronavirus disease 2019 (COVID-19) pandemic: an ACC/ SCAI consensus statement. JACC Cardiovasc Interv 2020; 13 : 1484-8.

63. Wood DA, Sathananthan J, Gin K, et al. Precautions and procedures for coronary and structural cardiac interventions during the COVID-19 pandemic: guidance from Canadian Association of Interventional Cardiology. Can J Cardiol 2020; 36: 780-3.

64. Chieffo A, Tarantini G, Naber CK, et al. Performing elective cardiac invasive procedures during the COVID-19 outbreak: a position statement from the European Association of Percutaneous Cardiovascular Interventions (EAPCI). Eurolntervention 2021; 16: 1177-86. 